\title{
Automatic Volumetry of the Cerebrospinal Fluid Space in Idiopathic Normal Pressure Hydrocephalus
}

\author{
Kazunari Ishii ${ }^{a, b}$ Tsutomu Somad, e Kenichi Shimadac Haruhiko Odac \\ Akira Terashimac Ryota Kawasaki ${ }^{b}$ \\ aDepartment of Radiology, Kinki University Faculty of Medicine, Osakasayama, \\ ${ }^{b}$ Department of Radiology, and ${ }^{\mathrm{C} I n s t i t u t e}$ for Aging Brain and Cognitive Disorders, Hyogo \\ Brain and Heart Center, Himeji, and d Department of Radiology, The University of Tokyo, and \\ e Software Development Group, FUJIFILM RI Pharma Co. Ltd., Tokyo, Japan
}

Key Words

Idiopathic normal pressure hydrocephalus · Volumetry · Cerebrospinal fluid space · MRI .

Ventricle system

\begin{abstract}
Objectives: To measure the cerebrospinal fluid (CSF) space volume in idiopathic normal pressure hydrocephalus (INPH), we developed a software that allows us to automatically measure the regional CSF space and compared the volumes of the ventricle systems (VS), Sylvian fissures (SF) and sulci at high convexity and midline (SHM) among INPH patients, Alzheimer's disease (AD) patients and healthy volunteers (HVs). Methods: Fifteen INPH patients, 15 AD patients and $15 \mathrm{HVs}$ were retrospectively selected for this study. 3D-T1 MR images were obtained. We improved upon an automatic gray matter volume system to measure CSF spaces, adopting new regions for the template of INPH-characteristic CSF spaces and measured them. The VS, SF and SHM volumes were calculated relative to the intracranial volume. Results: The relative SHM volume of the INPH group $(0.0237 \pm 0.0064)$ was the smallest among the 3 groups (AD: $0.0477 \pm 0.0109$, HV: $0.0542 \pm 0.0045)$. The VS $(0.0499 \pm 0.0135)$ and SF $(0.0187$ \pm 0.0037 ) volumes of the INPH group were significantly larger than those of the AD (VS: 0.0311 \pm 0.0075 , SF: $0.0146 \pm 0.0026$ ) and HV groups (VS: $0.0167 \pm 0.0065$, SF: $0.0111 \pm 0.017$ ). Conclusion: Automatic volume measurement can be used to delineate the characteristic changes in CSF space in patients with INPH and is useful in the diagnosis of INPH.
\end{abstract}




\section{Introduction}

Idiopathic normal pressure hydrocephalus (INPH) is a syndrome that is characterized by gait disturbance, mental deterioration and urinary incontinence, in association with normal cerebrospinal fluid (CSF) pressure. Its etiology remains unknown. The characteristic MRI findings in INPH include enlarged ventricle systems (VS) and Sylvian fissures (SF), and tight medial and high convexity sulci. Most cases of INPH exhibit these findings and are defined as disproportionately enlarged subarachnoid-space hydrocephalus (DESH) [1].

An Evans index $>0.3$ is necessary for the diagnosis of INPH. The callosal angle (CA) is also a useful index of the MRI finding of DESH and in discriminating INPH from other diseases [2]. However, the Evans index and CA are indices that indirectly express some components of DESH findings.

DESH findings include tight sulci at high convexity and medial subarachnoid spaces and enlarged SF with ventriculomegaly, defined as disproportionately enlarged subarachnoid space. However, there is no single characteristic index that allows physicians to quantitatively demonstrate these conditions. When considering these findings visually, there are some cases in which the judgment of the enlarged size and the degree of narrowing is difficult. Therefore, it is anticipated that the measurement of these CSF spaces would be simple and easy.

A voxel-based morphometry (VBM) method was used to investigate the characteristic regions of INPH [3], and Yamashita et al. [4] reported that the ratio of the CSF volume in the lateral ventricle/SF area to that in the high convexity/midline area in INPH patients could discriminate between INPH and patients with Alzheimer's disease (AD) and normal controls.

We improved upon an automatic brain volumetric software program (AVSIS), which we developed earlier [5-7], to automatically measure the volumes of the VS, SF, and medial and high convexity subarachnoid spaces in INPH, AD patients and healthy volunteers (HVs).

\section{Subjects and Methods}

\section{Subjects}

Fifteen consecutive patients with INPH, who had been admitted to our hospital for examination and treatment, were selected retrospectively from our institute's Dementia Registry. The patients had an MRI taken before a spinal tap test and fulfilled the criteria for diagnosis of probable INPH according to both the INPH guidelines [8] and the 'Guidelines for management of idiopathic normal pressure hydrocephalus: second edition' [9]. A spinal tap was performed and symptomatic improvement was confirmed in all patients.

The INPH group consisted of 6 males and 9 females. Their mean age was $78.4 \pm 6.2$ years, the mean Mini-Mental State Examination (MMSE) score was $23.1 \pm 4.6$, and the mean modified Rankin Scale score was $2.6 \pm 1.2$ (table 1). Comorbidities included gait disturbance in all 15, cognitive disorders in 14, and urinary incontinence in 11 patients. Fifteen patients with probable $\mathrm{AD}$, who had been diagnosed using the criteria from the National Institute of Neurological and Communicative Disorders and Stroke/Alzheimer's Disease and Related Disorders Association (NINCDS/ADRDA) [10], were also selected from our institute's Dementia Registry. The AD group also consisted of 6 males and 9 females. The mean age was $76.5 \pm 5.8$ years and the mean MMSE score was $22.1 \pm 5.7$. None of the patients in the AD group exhibited gait disturbance or urinary incontinence. Fifteen HVs were selected from our institute's Normal Registry. The group consisted on 8 males and 7 females. The mean age was $64.7 \pm 8.9$ years and the mean MMSE score was $29.1 \pm 1.2$. All study procedures were in agreement with the clinical study guidelines of the Ethics Committee of our institute. Written informed consent was waived for this study because it was retrospective in design. 
Table 1. Subject demographics

Ishii et al.: Automatic Volumetry of the Cerebrospinal Fluid Space in Idiopathic Normal Pressure Hydrocephalus

\begin{tabular}{llllll}
\hline Group & $\mathrm{n}$ & Sex (F:M) & Age, years & MMSE score & $\begin{array}{l}\text { mRS } \\
\text { score }\end{array}$ \\
\hline INPH & 15 & $9: 6$ & $78.4 \pm 6.2$ & $23.1 \pm 4.6$ & $2.6 \pm 1.2$ \\
AD & 15 & $9: 6$ & $76.5 \pm 5.8$ & $22.1 \pm 5.7$ & NA \\
HV & 15 & $8: 7$ & $64.7 \pm 8.9$ & $29.1 \pm 1.2$ & NA \\
\hline
\end{tabular}

mRS = Modified Rankin Scale; NA = not available

\section{MR Procedure}

We used 1.5-tesla Intera and 3.0-tesla Achieva MR scanners (both Philips Medical Systems, Best, The Netherlands). The images were taken with a 3D magnetization prepared rapid acquisition gradient echo (MPRAGE) imaging sequence. The nominal parameters of the MPRAGE were: sagittal plane, TR/TE/TI 2,400/3/1,000 ms, flip angle $8^{\circ}, 24 \mathrm{~cm} \mathrm{FOV,} 192 \times$ 192 in-plane matrix, 1.2-mm slice thickness.

\section{Volumetry}

All MRI data were transferred to a computer. Volumetry was performed with our AVSIS, which was improved for this study. In brief, the new AVSIS process is as follows: voxel of interest (VOI) templates for the intracranial, VS, SF, and the medial and high convexity subarachnoid space were prepared prior to this analysis. Each regional VOI template was produced on a digital phantom Simulated Brain Database (SBD; http://www.bic.mni.mcgill. $\mathrm{ca} /$ brainweb/) according to the standard Montreal Neurological Institute (MNI) space, with manual delineation of the contours of each structure (fig. $1 \mathrm{a}, \mathrm{b}$ ). The medial and high convexity subarachnoid space VOI template was produced manually, in reference to the results of a previous study of VBM [3] between INPH patients and HVs (fig. 1c). The process of this modified AVSIS is as follows: the MR image for each subject was segmented into gray matter (GM), white matter (WM), and CSF using the SPM8 segmentation program. The GM template image derived from the SBD was then spatially transformed into the GM image for each subject, and a normalization parameter was produced using SPM8 and the DARTEL technique. This normalization parameter functions in the same manner as a reverse parameter produced in the anatomical normalization of an individual brain to a standard brain. Using this parameter, the intracranial, hippocampal, VS, SF, and medial and high convexity subarachnoid space VOI templates were transformed to each individual subject's space. The intracranial volume (ICV) was adjusted using an image derived from the segmented GM, WM and CSF images. The segmented images were derived as follows: WM and GM areas were calculated with the voxels in the ICV VOI template. GM volumes in the hippocampi were calculated using the transformed hippocampal VOI templates. CSF volumes of the VS, SF, and sulci at high convexity and midline (SHM) were calculated using the transformed VS, SF, and SHM subarachnoid space VOI templates for each subject. Each regional relative volume was calculated by normalization to the ICV.

\section{Statistics}

The volume ratios of each structure in the 3 groups were compared with ANOVA and multiple group comparisons were performed. Correlations were evaluated between Evans index and CA and each relative volume of VS, SF and SHM subarachnoid space, and the hippocampus. The relative volumes of the VS, SF and SHM were tested as for their ability to distinguish between probable INPH and probable AD. A receiver-operating characteristic (ROC) analysis was performed to compare these relative volumes. 

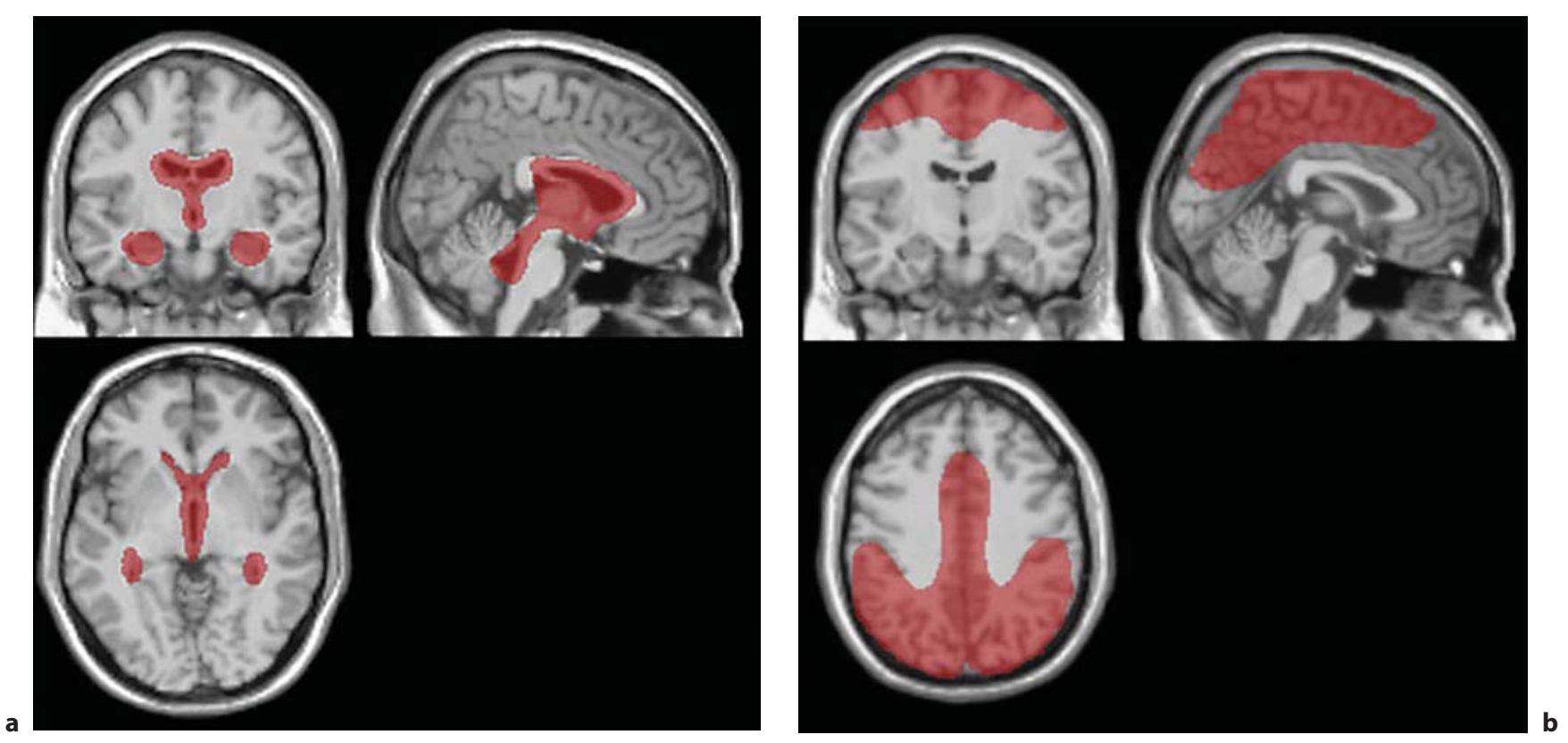

Fig. 1. VOI templates. a VOI template for CSF volume of ventricle systems. $\mathbf{b}$ VOI template for CSF volume of Sylvian fissures. c VOI template for CSF volume of sulci at high convexity and midline.

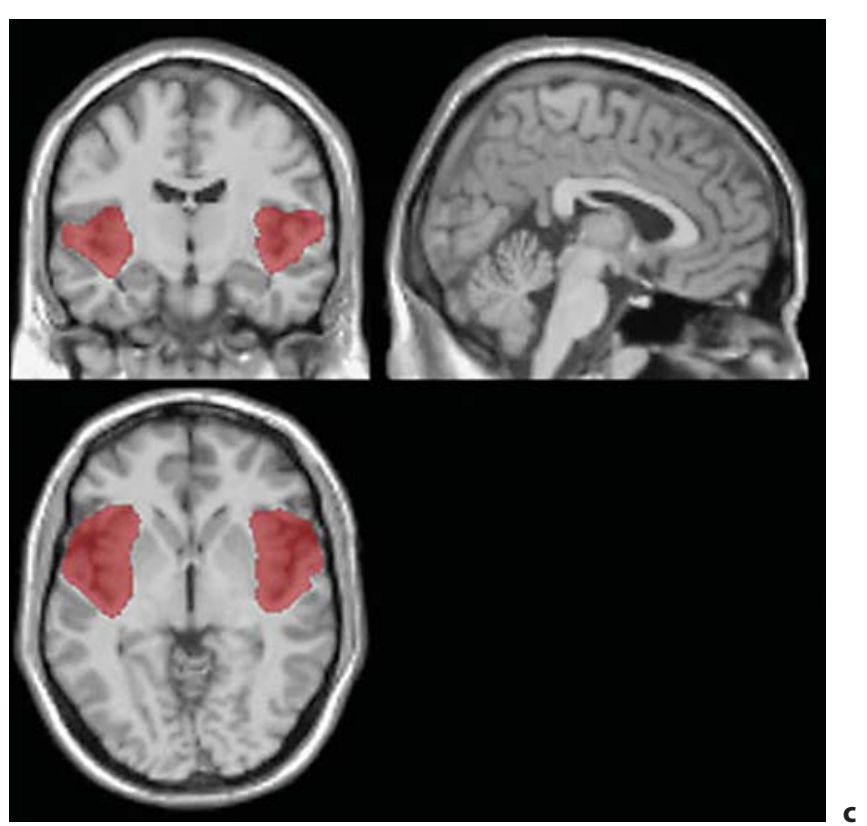

Results

Table 2 presents the mean Evans index and CA, and the mean ICV and volume of the whole brain (WB), hippocampus, VS, SF, and SHM subarachnoid space in the INPH, AD and HV groups. The CA of the INPH group was significantly smaller than those of the AD and HV groups. There were no differences between the ICV and WB of the 3 groups. The hippocampus of the AD group was significantly smaller than those of the INPH and HV groups. The volume of the cerebrospinal space at high convexity of INPH group was the smallest among the 3 groups. The volumes of the VS and SF of the INPH group were significantly larger than those of the AD and HV groups. 
Table 2. Evans index, $\mathrm{CA}\left({ }^{\circ}\right)$ and volumes $\left(\mathrm{mm}^{3}\right)$ of the structures of each group

\begin{tabular}{lllllllll}
\hline Group & Evans index CA & ICV & WB & Hip & SHM & VS & SF \\
\hline AD & $0.29 \pm 0.04$ & $107 \pm 14$ & $1,466 \pm 101$ & $1,033 \pm 73$ & $2.99 \pm 0.40^{*}$ & $69.8 \pm 16.0$ & $45.7 \pm 11.7^{\#}$ & $21.4 \pm 4.4$ \\
INPH & $0.34 \pm 0.03$ & $60 \pm 14^{*}$ & $1,512 \pm 108$ & $1,069 \pm 88$ & $3.33 \pm 0.36$ & $35.9 \pm 10.0^{*}$ & $75.2 \pm 19.7$ & $28.1 \pm 4.9^{\#}$ \\
HV & $0.24 \pm 0.03$ & $113 \pm 11$ & $1,451 \pm 51$ & $1,057 \pm 36$ & $3.43 \pm 0.27$ & $78.1 \pm 7.6$ & $23.7 \pm 11.0^{*}$ & $15.5 \pm 2.3^{*}$ \\
\hline
\end{tabular}

Hip $=$ Hippocampal volume. ${ }^{*}$ Significantly smaller than the other 2 groups $(\mathrm{p}<0.001) .{ }^{*}$ Significantly larger than the other 2 groups $(\mathrm{p}<0.001)$.

Table 3. Relative volumes of the structures of each group

\begin{tabular}{lllll}
\hline Group & Hip/ICV & SHM/ICV & VS/ICV & SF $/$ ICV \\
\hline AD & $0.0020 \pm 0.0002^{*}$ & $0.0477 \pm 0.0109$ & $0.0311 \pm 0.0075$ & $0.0146 \pm 0.0026$ \\
INPH & $0.0022 \pm 0.0001$ & $0.0237 \pm 0.0064^{*}$ & $0.0499 \pm 0.0135^{\#}$ & $0.0187 \pm 0.0037^{\#}$ \\
HV & $0.0023 \pm 0.0001$ & $0.0542 \pm 0.0045^{\#}$ & $0.0167 \pm 0.0065^{*}$ & $0.0111 \pm 0.017^{*}$ \\
\hline
\end{tabular}

Hip $=$ Hippocampal volume. ${ }^{*}$ Significantly smaller than the other 2 groups $(\mathrm{p}<0.001) .{ }^{\#}$ Significantly larger than the other 2 groups $(\mathrm{p}<0.001)$.

Table 4. Correlations of Evans index and CA with each volume

\begin{tabular}{llrl}
\hline & & $\mathrm{r}$ & $\mathrm{p}$ \\
\hline Evans index & VS/ICV & 0.724 & $<0.0001$ \\
& SF/ICV & 0.639 & $<0.0001$ \\
& SHM/ICV & -0.729 & $<0.0001$ \\
\hline $\mathrm{CA}$ & VS/ICV & -0.720 & $<0.0001$ \\
& SF/ICV & -0.644 & $<0.0001$ \\
& SHM/ICV & 0.854 & $<0.0001$ \\
\hline
\end{tabular}

Table 3 shows the relative hippocampal volume, and the relative volumes of the SHM, VS and SF normalized to ICV. The relative hippocampal volume of the AD group was significantly smaller than those of the other 2 groups. The relative volume of the SHM of the INPH group was the smallest among the 3 groups. The relative volumes of the VS and SF of the INPH group were the largest among the 3 groups.

The Evans index correlated well with the relative volume of the VS and SF, and it correlated inversely with the relative volume of the SHM. The CA also exhibited a good negative correlation with the relative volume of the VS and SF, and it correlated positively with the relative volume of the SHM (table 4).

Table 5 shows the area under the ROC curve for discriminating INPH from AD, based on the relative volume of VS, SF and SHM. The area under the ROC curve was the largest when based on the relative volume of the SHM, and it exhibited a very good performance in discriminating INPH patients from AD patients. 
Ishii et al.: Automatic Volumetry of the Cerebrospinal Fluid Space in Idiopathic Normal Pressure Hydrocephalus

Table 5. Areas under the ROC curves

\begin{tabular}{lllll}
\hline & $\begin{array}{l}\text { Area under the } \\
\text { ROC curve }\end{array}$ & Accuracy & Sensitivity & Specificity \\
\hline VS/ICV & 0.882 & $83 \%$ & $73 \%$ & $93 \%$ \\
SF/ICV & 0.816 & $80 \%$ & $87 \%$ & $73 \%$ \\
SHM/ICV & 0.982 & $97 \%$ & $100 \%$ & $93 \%$ \\
\hline
\end{tabular}

\section{Discussion}

The characteristic findings of INPH: (1) VS enlargement, (2) dilated SF and (3) tight sulci at high convexity were first reported by Kitagaki et al. [11], and these findings were confirmed by the voxel-based analysis [3]. Thus, INPH with these findings has been referred to as DESH. The Evans index can be used as an indirect index for detecting these findings, demonstrating an enlargement of the ventricles; however, there are currently no objective indices for dilated $\mathrm{SF}$ and tight sulci at medial parietal and high convexity. By visual inspection, there should be some variation between the observers in evaluating the widening of the SF and narrowing of the sulci.

The ratio of the CSF volume in the lateral ventricle/SF area to that in the high convexity/ midline area measured by the VBM method has been reported to be a good index for discriminating INPH from AD patients [4] and in monitoring the shunt responsiveness [12]. Because the template ROIs used in those studies were produced from the areas in which the CSF density of the INPH patients was significantly different from that of the AD patients or normal elderly controls, the ROIs did not cover the precise areas of the anatomically defined VS, SF, or SHM, and the method could not be used to measure the absolute values of each structure. Therefore, in order to measure the absolute values of those areas, we developed an automatic measuring system and obtained reasonable findings. As we have produced a program that automatically measures the volume of brain structures, we improved this system to allow us to measure CSF spaces.

A previous study reported on SIENA, a tool from the FSL software library, it automatically measures the global brain volume, but the volume of the ventricles was measured by manual segmentation [13]. Ambraki et al. [14] evaluated a commercially available automatic software for measuring ICV, brain tissue and ventricular volume. However, ICV was measured on T2-weighted images, and brain tissue volume and ventricular volume were measured on FLAIR images with $3 \mathrm{~mm}$ thickness and $0.5 \mathrm{~mm}$ intersectional gaps, which were not 3D images. The semi-automated software Brain Ventricle Quantification (BVQ) is available, but it requires operator-selected seed points in each lateral ventricle, and a region-growing algorithm automatically expanded the seed points within the 3D space of the image to the margin of the periventricular tissue. This does notallow one to measure the volume of the subarachnoid CSF space [15].

Lebret et al. [16] reported on a new automatic method to measure the volumes of the CSF spaces for the diagnosis of hydrocephalus through segmentation and separation steps, which implements image properties, anatomical and geometrical features as well as topological assumptions of the entire fluid shape. However, this method requires another whole-body MRI sequence that significantly highlights the CSF. Our method, on the other hand, requires no new MRI sequence(s) but uses previously scanned 3D T1-weighted images (e.g. MPRAGE).

The results of this study met our expectations: the VS and FS volumes of the INPH patients were significantly larger than those of the AD patients, and the SHM volume of the INPH patients was significantly less than that of the AD patients, which is precisely consistent with 
Ishii et al.: Automatic Volumetry of the Cerebrospinal Fluid Space in Idiopathic Normal Pressure Hydrocephalus

the DESH findings. Thus, the measurement of these volumes will allow a discrimination of INPH patients from brain atrophy diseases, such as AD.

A previous report indicated a very strong correlation ( $r$ : 0.95) between the Evans index and ventricle volumes [17]; thus, while our findings are good ( $\mathrm{r}$ : 0.76), they are not as good as theirs. However, our findings are compatible with the previous report, as the Evans index demonstrated an enlargement of the anterior horn of the lateral ventricles, but not of the whole volume of the ventricles. The CA also correlated well with the enlargement of SF and VS, and the correlation coefficient between CA and SHM volume was the largest ( $\mathrm{r}$ : 0.854). This means that tight sulci, especially at the midline, lead to a sharp CA and that tight SHM are probably due to a compression by the enlarged SF and VS. Therefore, the correlation coefficients between the CA, SF and VS were not as good as those of the SHM volume.

Measuring the SHM volume and demonstrating a smaller SHM volume is very useful in discriminating INPH patients from AD patients. The area under the ROC curve of SHM was larger than the others and the accuracy was the highest. A small SHM volume manifests with tight SHM. This finding reflects the DESH finding in INPH. In AD patients, the VS and SF volumes tend to increase due to brain atrophy; therefore, the areas under the ROC curves of VS and SF are inferior to that of SHM.

One limitation of this study is that this was a retrospective study that was performed at a single institution. Further multicenter studies are necessary, and the usefulness of this automatic volumetry software should be verified.

\section{Conclusion}

We further developed an automatic measurement software for INPH that demonstrates the characteristic CSF volumes and obtains compatible findings. This approach supports the diagnosis of INPH and will aid in further research of the pathophysiology of INPH.

\section{References}

1 Hashimoto M, Ishikawa M, Mori E, Kuwana N; Study of INPH on neurological improvement (SINPHONI): Diagnosis of idiopathic normal pressure hydrocephalus is supported by MRI-based scheme: a prospective cohort study. Cerebrospinal Fluid Res 2010;7:18.

-2 Ishii K, Kanda T, Harada A, Miyamoto N, Kawaguchi T, Shimada K, Ohkawa S, Uemura T, Yoshikawa T, Mori E: Clinical impact of the callosal angle in the diagnosis of idiopathic normal pressure hydrocephalus. Eur Radiol 2008;18:2678-2683.

-3 Ishii K, Kawaguchi T, Shimada K, Ohkawa S, Miyamoto N, Kanda T, Uemura T, Yoshikawa T, Mori E: Voxelbased analysis of gray matter and CSF space in idiopathic normal pressure hydrocephalus. Dement Geriatr Cogn Disord 2008;25:329-335.

4 Yamashita F, Sasaki M, Takahashi S, Matsuda H, Kudo K, Narumi S, Terayama Y, Asada T: Detection of changes in cerebrospinal fluid space in idiopathic normal pressure hydrocephalus using voxel-based morphometry. Neuroradiology 2010;52:381-386.

-5 Ishii K, Soma T, Kono AK, Sofue K, Miyamoto N, Yoshikawa T, Mori E, Murase K: Comparison of regional brain volume and glucose metabolism between patients with mild dementia with lewy bodies and those with mild Alzheimer's disease. J Nucl Med 2007;48:704-711.

-6 Ishii K, Soma T, Kono AK, Sasaki H, Miyamoto N, Fukuda T, Murase K: Automatic volumetric measurement of segmented brain structures on magnetic resonance imaging. Radiat Med 2006;24:422-430.

-7 Goto H, Ishii K, Uemura T, Miyamoto N, Yoshikawa T, Shimada K, Ohkawa S: Differential diagnosis of dementia with Lewy Bodies and Alzheimer Disease using combined MR imaging and brain perfusion single-photon emission tomography. AJNR Am J Neuroradiol 2010;31:720-725.

8 Relkin N, Marmarou A, Klinge P, Bergsneider M, Black PM: Diagnosing idiopathic normal-pressure hydrocephalus. Neurosurgery 2005;57:S4-S16; discussion ii-v.

-9 Mori E, Ishikawa M, Kato T, Kazui H, Miyake H, Miyajima M, Nakajima M, Hashimoto M, Kuriyama N, Tokuda T, Ishii K, Kaijima M, Hirata Y, Saito M, Arai H: Guidelines for management of idiopathic normal pressure hydrocephalus: second edition. Neurol Med Chir (Tokyo) 2012;52:775-809. 
10 McKhann G, Drachman D, Folstein M, Katzman R, Price D, Stadlan EM: Clinical diagnosis of Alzheimer's disease: report of the NINCDS-ADRDA Work Group under the auspices of Department of Health and Human Services Task Force on Alzheimer's Disease. Neurology 1984;34:939-944.

11 Kitagaki H, Mori E, Ishii K, Yamaji S, Hirono N, Imamura T: CSF spaces in idiopathic normal pressure hydrocephalus: morphology and volumetry. AJNR Am J Neuroradiol 1998;19:1277-1284.

-12 Wada T, Kazui H, Yamamoto D, Nomura K, Sugiyama H, Shimizu Y, Yoshida T, Yoshiyama K, Yamashita F, Kishima H, Yoshimine T, Takeda M: Reversibility of brain morphology after shunt operations and preoperative clinical symptoms in patients with idiopathic normal pressure hydrocephalus. Psychogeriatrics 2013;13: 41-48.

13 Singer OC, Melber J, Hattingen E, Jurcoane A, Keil F, Neumann-Haefelin T, Klein JC: MR volumetric changes after diagnostic CSF removal in normal pressure hydrocephalus. J Neurol 2012;259:2440-2446.

14 Ambarki K, Wahlin A, Birgander R, Eklund A, Malm J: MR imaging of brain volumes: evaluation of a fully automatic software. AJNR Am J Neuroradiol 2011;32:408-412.

15 Nestor SM, Rupsingh R, Borrie M, Smith M, Accomazzi V, Wells JL, Fogarty J, Bartha R; Alzheimer's Disease Neuroimaging Initiative: Ventricular enlargement as a possible measure of Alzheimer's disease progression validated using the Alzheimer's disease neuroimaging initiative database. Brain 2008;131:2443-2454.

-16 Lebret A, Hodel J, Rahmouni A, Decq P, Petit E: Cerebrospinal fluid volume analysis for hydrocephalus diagnosis and clinical research. Comput Med Imaging Graph 2013;37:224-233.

17 Ambarki K, Israelsson H, Wahlin A, Birgander R, Eklund A, Malm J: Brain ventricular size in healthy elderly: comparison between Evans index and volume measurement. Neurosurgery 2010;67:94-99; discussion 99. 